\title{
Agriculture et changement climatique : un mariage de raison inéluctable
}

\section{Agriculture and climate change: an unavoidable marriage of convenience}

Depuis décembre 2015 et l'organisation à Paris de la $21^{\mathrm{e}}$ conférence des parties de la Convention-cadre des Nations unies sur les changements climatiques (COP 21, CCNUCC), la planète climat n'est plus tout à fait la même. L'accord international sur le climat signé lors de cette conférence, fixant comme objectif une limitation du réchauffement mondial entre $1,5^{\circ} \mathrm{C}$ et $2{ }^{\circ} \mathrm{C}$ d'ici 2100 , est un évènement majeur. L'histoire dira si le monde est capable de tenir cet engagement, mais le cinquième rapport du Groupe d'experts intergouvernemental sur l'évolution du climat (GIEC), paru en 2014, qui prévoit une hausse des températures de 0,3 à $4,8^{\circ} \mathrm{C}$ d'ici 2100 , est lui sans appel. Personne ne pourra dire « on ne savait pas ».

En France, les scientifiques s'étaient mobilisés bien avant la COP 21 sur les questions climatiques. Dans le secteur de l'usage des terres notamment, une Conférence internationale sur l'agriculture « intelligente » face au climat (Climate-smart agriculture) avait été organisée à Montpellier en mars 2015, réunissant plus de 750 participants venus de 75 pays. En juillet 2015 à Paris, c'est la conférence «Our Common Future Under Climate Change» qui réunissait plus de 2200 participants de 100 pays. Lors de ces évènements importants, les participants ont fait des propositions ayant pour but d'alimenter les débats de la COP 21 et ainsi de permettre que soit signé un accord bien informé. L'apparition de la sécurité alimentaire dans l'accord de Paris n'est sans doute pas étrangère à cette mobilisation (alors que les négociations climatiques dans le secteur de l'usage des terres peinaient jusque-là à reconnaître l'importance de l'agriculture et ciblaient essentiellement les forêts). En 2017, lors de la COP 23, la boucle est bouclée avec l'adoption d'une recommandation sur la vulnérabilité de l'agriculture face au changement climatique et la prise en compte de la sécurité alimentaire. Cette recommandation cite notamment l'adaptation de l'agriculture, la résilience, le rôle du carbone du sol, l'importance des nutriments, la place de l'élevage et les dimensions socio-économiques de ces approches.

Le monde agricole se trouve ainsi au cœur d'une partie de billard à 3 bandes : l'agriculture subit les effets du changement climatique, souvent de manière dramatique, notamment dans les pays tropicaux; l'agriculture est en partie responsable du changement climatique ( $24 \%$ des émissions de gaz à effet de serre, en incluant les changements d'usage des terres qui sont liés) ; l'agriculture peut contribuer à atténuer le changement climatique en fixant du carbone dans le sol et la biomasse. Sur ce dernier point, les chiffres montrent qu'une augmentation moyenne annuelle de $4 \%$ ( 4 pour mille, ou 0,4\%) du taux de carbone de tous les sols de la planète pourrait compenser les émissions de gaz à effet de serre dues à l'homme. Lancé comme un défi aux chercheurs lors de la conférence «Climate-Smart Agriculture» de mars 2015 à Montpellier, ce taux de 4 pour 1000 est devenu un slogan et définit un cap à suivre. L'Initiative « 4 pour 1000 » sera officiellement lancée lors de la COP 21 à Paris, puis prendra forme avec une gouvernance spécifique lors de la COP 22 de Marrakech. Un consortium des membres (149 adhésions fin 2017) définit des orientations et est adossé à un forum (281 membres fin 2017), qui réunit tous types de parties prenantes des secteurs publics et privés. Un conseil scientifique et technique regroupant des chercheurs du monde entier examine les projets de l'initiative.

À l'origine de la conférence de mars 2015, le concept de «Climate-Smart Agriculture», perçu par certains comme l'explicitation d'un cadre permettant de traiter les questions entre agriculture et climat, par d'autres comme lié aux agro-industries et vecteur de comportements opportunistes, fait l'objet de nombreux débats. Son utilité est conditionnée par la capacité à dépasser une approche exclusivement techniciste et à intégrer les dimensions sociales, territoriales et écologiques des transformations en cours, mais surtout à constituer un cadre renouvelé d'évaluation des performances des pratiques agricoles. De son côté, l'importance du carbone du sol semble faire l'unanimité et s'est donc révélé mobilisatrice. Il n'empêche que le slogan « 4 pour mille» soulève de nombreuses questions. Elles portent tout d'abord sur la prise en compte des dynamiques de stockage dans la durée ou encore sur les potentiels et dynamiques différenciés d'un lieu à l'autre. Elles invitent en outre à ne pas faire l'impasse sur les émissions d'oxyde nitreux $\left(\mathrm{N}_{2} \mathrm{O}\right)$ et de méthane $\left(\mathrm{CH}_{4}\right)$ et sur les liens directs et indirects entre ces émissions et le stockage de carbone. Toutes les approches doivent en outre accorder une attention particulière à l'adaptation au changement climatique, dès lors qu'il est question d'atténuation, pour recevoir un accueil favorable de la part des agriculteurs, notamment ceux du Sud.

Les articles présentés dans le numéro thématique des Cahiers Agricultures «Les agricultures face au changement climatique » reflètent l'évolution de ces débats sur les liens entre agriculture et changement climatique depuis 2015, en lien avec les évolutions des injonctions et de l'agenda politiques. Les auteurs montrent que le changement climatique pose des questions inédites 
auxquelles les agriculteurs répondent de manière différenciée. En Afrique subsaharienne, à Madagascar, en Thaïlande ou en France, les innovations visant l'adaptation sont possibles et diverses. Impliquant les choix technologiques, la gestion des exploitations, les institutions collectives locales, ces processus questionnent également la capacité des politiques publiques à stimuler et accompagner l'innovation et à anticiper risques et aléas. C'est bien en agissant de manière cohérente à différents niveaux d'organisation que ces processus pourront se traduire par un impact significatif. Cet enjeu de l'impact soulève bien évidemment de nombreuses questions - et donc des défis scientifiques -, qu'il s'agisse de saisir les trajectoires de transformation sur le long terme, d'en rendre compte par des indicateurs pertinents et mesurables, ou d'en qualifier et quantifier les tenants et aboutissants en lien avec les objectifs du développement durable.

Dans tous les cas, nous savons que de nouveaux modes de gouvernance sont nécessaires pour que la crise climatique ne se transforme pas en catastrophe et pour mobiliser dans cette perspective la connaissance intime des systèmes agricoles par les agriculteurs du monde et stimuler l'innovation. La santé de la planète et la sécurité alimentaire mondiale en dépendent.

Que ce soit ici ou là-bas, la science est appelée à jouer un rôle essentiel. Au-delà de l'invention technologique, il lui revient de saisir les processus en cours, humains comme climatiques, et d'éclairer l'incertain et les futurs possibles. Il nous revient ensemble d'inventer de nouvelles formes de dialogue et d'interaction pour mieux fonder la décision et ainsi éviter le pire.

Ministre de l'Agriculture, 2012-2017; Porte-parole du Gouvernement, 2014-2017, Député de la Sarthe, Vice-Président de l'Initiative " 4 pour 1000 Les sols pour la sécurité alimentaire et le climat»

Patrick Caron ${ }^{1,3}$

Directeur Général délégué à la Recherche et à la Stratégie du Cirad, 2010-2016

Emmanuel Torquebiau, ${ }^{2,3}, *$

Chercheur au Cirad, Chargé de mission changement climatique, 2012-2017, Coordonnateur du numéro thématique des Cahiers Agricultures "Les agricultures face au changement climatique»

${ }^{1}$ CIRAD, DGDRS, 34398 Montpellier, France ${ }^{2}$ CIRAD, UPR AIDA, 34398 Montpellier, France

${ }^{3}$ Univ Montpellier, CIRAD, Montpellier, France

*emmanuel.torquebiau@cirad.fr 